\title{
Molecular Substrates of Social Avoidance Seen following Prenatal Ethanol Exposure and Its Reversal by Social Enrichment
}

\author{
Frank A. Middleton ${ }^{a, b}$ Elena I. Varlinskaya ${ }^{b, c}$ Sandra M. Mooney ${ }^{b, d}$ \\ ${ }^{a}$ Department of Neuroscience and Physiology, SUNY Upstate Medical University, Syracuse, N.Y., ${ }^{b}$ Developmental \\ Exposure Alcohol Research Center, Binghamton, N.Y., Cortland, N.Y., Syracuse, N.Y., Baltimore, Md., ' Department of \\ Psychology, Center for Development and Behavioral Neuroscience, Binghamton University, Binghamton, N.Y., and \\ ${ }^{\mathrm{d}}$ Department of Pediatrics, University of Maryland School of Medicine, Baltimore, Md., USA
}

\section{Key Words \\ Ethanol · Autism • Fetal alcohol syndrome $\cdot$ Behavior • \\ Microarray $\cdot$ Amygdala $\cdot$ Basal ganglia}

\begin{abstract}
Prenatal ethanol exposure is associated with, and is a risk factor for, developmental disorders with abnormal social behaviors, including autism spectrum disorders. We hypothesize that the specific effects of ethanol on social behavior are defined by the timing of the exposure as well as subsequent changes in brain regions such as the amygdala and ventral striatum. We recently reported that in utero ethanol exposure on gestational day 12 alters social behaviors of weanling [postnatal day (P) 28], adolescent (P42), and young adult (P75) rats. Male, but not female, offspring of the ethanol-exposed dams showed significant decreases in social investigation (sniffing of a social partner), contact behavior (grooming or crawling over/under the partner), and play fighting (following, chasing, nape attacks, or pinning) at all ages tested with maximal effects at P28 and P42. Furthermore, ethanol-exposed males and females showed evidence of social avoidance at P42 and P75. The present study sought to test whether a form of social enrichment could normalize any of
\end{abstract}

the social deficits and what the molecular mechanisms of such effects might be. We found that housing rats with nonmanipulated control rats normalized the social avoidance phenotype normally seen when they are housed with sexmatched prenatal ethanol-exposed littermates. There was no mitigation of the other ethanol-induced behavioral deficits. Conversely, male control-treated rats housed with nonlittermates showed deficits in play fighting, social investigation and contact behavior. Molecular analyses of the amygdala and ventral striatum of adolescent rats following fetal ethanol exposure indicated several specific neurotransmitter systems and pathways that might underlie the social avoidance phenotype as well as its reversal.

Copyright $\odot 2012$ S. Karger AG, Basel

\section{Introduction}

Social behavior deficits are a feature of a number of developmental disorders, including fetal alcohol spectrum disorder and autism. It should be noted that social behavioral deficits are rarely uniform within a diagnostic group, let alone among different developmental disorders. Nonetheless, these deficits can have major negative

\section{KARGER}

Fax +4161306 1234

E-Mail karger@karger.ch

www.karger.com
C 2012 S. Karger AG, Basel

$0378-5866 / 12 / 0343-0115 \$ 38.00 / 0$

Accessible online at:

www.karger.com/dne
Sandra M. Mooney, $\mathrm{PhD}$

Department of Pediatrics, University of Maryland School of Medicine 655 W. Baltimore St, BRB 13-041

Baltimore, MD 21201 (USA)

Tel. +1 410706 2615, E-Mail smooney@ peds.umaryland.edu 
effects on quality of life. A recent report on people with autism shows that subjects with mild social behavior deficits display the same quality of life issues as those with more severe deficits [1]. Thus, even mild deficits in social behavior should be taken seriously and mitigated if at all possible. To date, the best intervention for social behavior deficits is early intensive behavioral therapy but this is time-consuming and costly.

In humans with fetal alcohol spectrum disorder, social behavior abnormalities include deficits in relationships, coping skills, and use of play and leisure time $[2,3]$. Other problems include poor social skills, difficulties understanding social cues, and inappropriate social behavior $[4,5]$. Such deficits continue through adolescence and into adulthood [6].

Rats exposed to alcohol during development also have altered social behaviors with the behavioral changes being dependent on the timing, duration, and dose of alcohol. Social play is affected in male rats exposed to ethanol during the second half of gestation [7], whereas social interactions were mostly increased in both males and females exposed to alcohol throughout gestation to postnatal day (P) 10 [8]. We recently reported that acute exposure to ethanol on a single day of gestation affects social behavior in a way that is timing-, age-, and sex-dependent [9]. That is, male offspring of animals exposed on gestational day (G) 12 exhibit deficits in play fighting, contact behavior, and social investigation during adolescence and adulthood.

Social behavior normally relies on multiple sensory systems (vision, olfaction, audition, and somatosensation), as well as on the limbic system. Within the limbic system the amygdala is widely recognized as being particularly important for social behavior. Lesions in the amygdala result in aberrant social behaviors and reduced eye contact behavior [10-12]. Moreover, people with amygdala damage do not process emotional information normally [13-15]. Notably, these symptoms are similar to those of people with autism.

The anatomical substrates for amygdala involvement in social behavior are beginning to be elucidated. Price [16] describes amygdala connectivity as having three main systems: forebrain-sensory, forebrain-emotional, and brainstem. The central nucleus of the amygdala is a key player in the forebrain-emotional system. This system involves connections between numerous brain areas including the prefrontal cortex, medial thalamus, and basal ganglia, and appears to be important for regulating mood, behavioral choices, reward assessment and modulation of visceral function. Within the basal ganglia, it is thought that the ventral striatum serves as an integration center for social/emotional signals from the amygdala, spatial/contextual information from the hippocampus, reward/motivational signals from midbrain dopamine neurons, and cognitive signals from prefrontal areas of the cortex (reviewed in Pennartz et al. [17]). Once integrated, the information is thought to guide the selection or execution of appropriate behavioral responses to different cues.

Social dysfunction may be the most characteristic feature of autism. Therefore, improved social functioning has often been one of the main goals of intervention. A number of social intervention strategies for children and adults have been designed and tested in behavioral studies. Child-parent social interactions [18], child-other adult social interactions $[19,20]$, and child-peer social interactions [21] can be effectively taught using these behavioral methods. It has been demonstrated that social skills, when trained, can also improve verbal and nonverbal communication [22]. Social characteristics of the environment play an important role in social skills training, with a greater ratio of typically developing peers to atypically developing peers (i.e. autistic peers) supporting improved social interactions for peers with autism [23]. Therefore, social experience with typically developing peers is essential for improving social skills and increasing social interactions in autistic children.

The beneficial effects of social enrichment can also be seen in animal models. Socially deprived animals exhibit behavioral deficits similar to some of those seen in autism. Specifically they show repetitive, or stereotyped, behaviors following CNS damage, administration of pharmacological agents, or environmental restriction [24]. Subsequently, many of these behavioral deficits can be ameliorated by housing in enriched environments. Environmental enrichment also ameliorates the deficits in social behavior caused by prenatal exposure to valproic acid [25], a commonly used model of autism. Behavioral studies have revealed substantial effects of enriched environments on learning and memory [26-28], anxiety-related behaviors [29-32] and exploratory activity [27, 33]. Intriguingly, social factors are associated with changes in novelty responsiveness and anxiety-like behavior by affecting the prefrontal cortex and striatum [34-37].

We have previously shown that acute exposure to ethanol on G12 negatively affected four measures of social behavior in male offspring (investigation, contact, play fighting, and social motivation), whereas females only showed deficits in social motivation [9]. Interestingly, with increasing age, both males and females became 
more avoidant of a novel conspecific. Given that social factors can moderate anxiety behaviors, we tested the hypothesis that social enrichment could ameliorate the behavioral deficits caused by acute exposure to ethanol during mid-gestation. We also sought to determine the molecular mechanisms that contribute to this improvement in social functioning.

\section{Methods}

\section{Animals}

Pregnant Long Evans rats (Harlan, Indianapolis, Ind., USA) were received on G4. G1 was designated as the first day on which a sperm-positive plug was noted. Rats were housed at the Syracuse Veteran's Affairs Medical Center in a facility accredited by the Association for Assessment and Accreditation of Laboratory Animal Care (AAALAC). Rooms were temperature-controlled $\left(22^{\circ} \mathrm{C}\right)$ and maintained on a 12 -hour light/12-hour dark cycle (lights off at 07:00). All procedures were performed with approval of the Committee for Humane Use of Animals at SUNY Upstate Medical University and the Institutional Animal Care and Use Committee at the Syracuse Veteran's Affairs Medical Center, and were in accordance with the guidelines for animal care established by the National Institutes of Health.

Animals were exposed to ethanol prenatally as described previously [9]. Briefly, on G12, dams received an intraperitoneal injection of ethanol $(2.9 \mathrm{~g} / \mathrm{kg}$ as a $20 \% \mathrm{v} / \mathrm{v}$ solution in physiological saline) at time $0\left(\mathrm{t}_{0}\right)$. Two hours later $\left(\mathrm{t}_{2}\right)$, animals received a second intraperitoneal injection of $1.45 \mathrm{~g} / \mathrm{kg}$ ethanol. Control animals received an intraperitoneal injection of an equivalent volume of saline at $t_{0}$ and again at $t_{2}$. This method of ethanol administration gives blood ethanol concentrations of $287 \pm 3.5 \mathrm{mg} / \mathrm{dl}$ within $15 \mathrm{~min}$ of the second injection [9].

Litters were culled to 10 pups within $24 \mathrm{~h}$ of birth, maintaining a 50/50 ratio of males/females as best as possible. On P21, litters were weaned and male and female offspring were housed separately. After social behavior testing (described below), animals were injected intraperitoneally with $100 \mathrm{mg} / \mathrm{kg}$ ketamine and 10 $\mathrm{mg} / \mathrm{kg}$ xylazine prior to decapitation. Brains were rapidly removed, snap-frozen in dry ice, and stored at $-80^{\circ} \mathrm{C}$ until used for RNA extraction (below).

\section{Social Enrichment}

For this study, we defined social enrichment as housing experimental animals (offspring of saline- or ethanol-injected dams) with novel conspecifics from untreated dams for 3 weeks between P21 and P42. All animals were housed in groups of 3 or 4. Thus, nonenriched animals were housed in groups of 3 or 4 same-sex littermates between P21 and P42. For enriched animals, 1 offspring from an experimental dam (saline- or ethanol-treated) was housed with 2 or 3 same-age, same-sex animals from a nontreated dam between P21 and P42.

\section{Social Interaction Test}

On P42, animals underwent a modified social interaction test as described previously [9]. All testing was conducted under dim light using a Plexiglas test apparatus $(30 \mathrm{~cm} \times 20 \mathrm{~cm} \times 20 \mathrm{~cm})$ divided into two equally sized compartments by a clear partition with an aperture $(7 \mathrm{~cm} \times 5 \mathrm{~cm})$ that allowed 1 animal at a time to move between compartments.

On P41, the day before testing, experimental animals were placed into the testing apparatus by themselves for $30 \mathrm{~min}$. This made the test environment more familiar, thereby increasing social interactions $[38,39]$. On P42, each experimental animal was marked on its side with permanent ink, placed in a holding cage alone for $30 \mathrm{~min}$ and then transferred into the test apparatus. After $5 \mathrm{~min}$, a novel age- and sex-matched control rat from a nontreated dam was also placed into the testing apparatus.

Animals were recorded for the 10 -min social interaction test session. Behavioral data were scored from video recordings by a trained observer who was blinded to the prenatal treatment, sex, and postnatal housing of any animal. Five behavioral measures were scored: play fighting, social investigation, contact behavior, social motivation, and locomotor activity. Social investigation was defined as the sniffing of any part of the body of the cage partner. Contact behavior was defined as crawling over and under the partner and social grooming. Play fighting was defined as pouncing or playful nape attack, following and chasing, and pinning. Social motivation was determined by counting the number of crossovers (movements between compartments) toward the partner and the number of crossovers away from the partner. Social motivation was expressed as a coefficient of social preference/ avoidance [coefficient $(\%)=($ crossovers to - crossovers from $) /$ (crossovers to + crossovers from)]

\section{Statistical Analysis of Behavior}

The design of the study was a 2 (prenatal exposure: saline or ethanol) $\times 2$ (postnatal housing: nonenriched or enriched) $\times 2$ (sex) factorial, with 9-12 experimental subjects placed into each of the 8 groups defined by this factorial design. Data were analyzed separately for each behavioral measure using a corresponding $2 \times 2 \times 2$ analysis of variance (ANOVA). Fisher's least significant difference tests were used for post hoc comparisons to determine the source of any significant main effects and interactions.

\section{RNA Isolation}

To determine the potential molecular substrates of increased and decreased social motivation, we first dissected the whole amygdala and ventral striatum from a total of 7242 -day-old male and female rats, using established anatomical landmarks. For these dissections, the brains were first cut into coronal slabs. The boundaries of the amygdala complex (putamen dorsally, piriform cortex laterally, and internal capsule medially) were visualized on the first coronal slab caudal to the optic chiasm that contained clear thalamus and hippocampus. The boundaries of the ventral striatum were visualized on the most rostral coronal slab containing clear striatum and lateral ventricles rostral to the corpus callosum. The ventral striatum was defined as the subcortical brain region ventral and medial to a line starting at the ventral limit of the lateral ventricle extending $45^{\circ}$ below the horizontal plane toward the ventrolateral cortical surface. After isolating the regions of interest, total RNA was purified using the RNeasy kit (Qiagen, Valencia, Calif., USA). RNA yield and quality were assessed by UV spectrophotometry and the Agilent Technologies Bioanalyzer. 


\section{Expression Profiling}

For each of the 8 conditions in the $2 \times 2 \times 2$ design, we used 3 Rat ST Gene 1.0 Arrays (Affymetrix, Santa Clara, Calif., USA) to examine changes in gene expression in 2 different brain regions (the amygdala and ventral striatum) for a total of 48 microarrays. Each array was hybridized with a pooled RNA sample prepared from 3 separate rats within the same condition (100 ng total RNA per rat sample), and 3 pools were used for each condition. RNA was prepared for hybridization using the WT Expression Kit (Ambion, Austin, Tes., USA). After hybridization, washing and scanning, the 48 arrays were normalized using GC-RMA and imported into Partek Genomics Suite for analysis (version 6.5). For this report, we used the NetAffx query tool (Affymetrix) to identify and limit our analysis to a total of 663 probe sets on the Rat ST Gene 1.0 Array that were involved in brain and relevant behavioral functions using the following 17 gene ontology search terms: social, anxiety, fear, autism, synapse, synaptic, norepinephrine, neuropeptide, cannabinoid, opioid, oxytocin, GABA, glutamate, glycine, serotonin, dopamine, neurotransmitter.

\section{Molecular Substrates of Decreased Social Motivation}

Because the behavioral data indicated that social motivation was significantly decreased in both male and female rats that were exposed in utero to ethanol at G12, we first compared the in utero exposed versus unexposed $\mathrm{P} 42$ rats in both brain areas. This was done using a $2 \times 2 \times 2$ (exposure $\times$ sex $\times$ brain area) ANOVA, with post hoc contrasts set to reveal the change due to prenatal ethanol exposure. We focused on those genes with a significant main effect of the ethanol exposure after correction for multiple testing (using a step-up false discovery rate algorithm) and lack of any significant ethanol exposure $x$ sex or ethanol exposure $\times$ area interactions. For the genes that met these criteria, we also determined the evidence for any social enrichment $\times$ ethanol interactions.

\section{Molecular Substrates of Increased Social Motivation}

Because the behavioral data demonstrated that enrichment with a normal cagemate reversed the social motivation deficit seen in prenatal ethanol-exposed male and female rats, we next compared the enriched versus nonenriched ethanol-exposed rats. Similar to the previous analysis, this was performed using a $2 \times$ $2 \times 2$ (enrichment condition $\times$ sex $\times$ brain area) ANOVA, with post hoc contrasts set to reveal the change due to social enrichment. We focused on those genes with a significant main effect of the enrichment after correction for multiple testing (using a stepup false discovery rate algorithm) and lack of any significant enrichment $\times$ sex or enrichment $x$ area interactions. For the genes that met these criteria, we also determined the evidence for any social enrichment $x$ ethanol interactions.

\section{Clustering, Bioinformatic, and Alternative Splice Variant}

Analyses

Genes with significant main effects of either gestational ethanol exposure or social enrichment were subjected to hierarchical cluster analysis and annotated using the STRING database (http:// string-db.org). Then, we used STRING to construct a biological interaction network using the 28 unique genes, plus twice that number $(\mathrm{n}=56)$ of connecting nodes (genes not in the list). Only interactions with high confidence ( 0.7 score or greater) were used. Finally, we used the Partek Genomics Suite alternative splice vari- ant (ASV) algorithm to determine if any of the observed changes in overall gene expression seen as a result of ethanol exposure or social enrichment might be explained or influenced by different patterns of exon usage in the treatment groups. This algorithm tests all of the exon probes (approximately 9-10 per gene) within the genes of interest, using the gene identity as an additional factor in the ANOVA model, thus enabling the detection of exon probe $\times$ treatment interactions within each gene. Such interactions indicate potentially different patterns of exon usage (i.e., ASVs) that are related to treatment variables, such as ethanol exposure or social enrichment. Only genes with 3 or more exons were examined by this algorithm, and multiple testing correction was applied.

\section{Results}

\section{Social Behavior}

Prenatal exposure to ethanol on G12 altered social behavior in 42-day-old rats (fig. 1), with positive consequences of social enrichment evident in animals prenatally exposed to ethanol and negative effects seen in saline-exposed males.

For each behavioral measure, separate three-way ANOVAs using a 2 (prenatal exposure: saline or ethanol) $\times 2$ (postnatal housing: nonenriched or enriched) $\times 2$ (sex) factorial design revealed significant three-way interactions for social investigation $[\mathrm{F}(1,74)=4.26, \mathrm{p}<$ $0.05]$ and play fighting $[\mathrm{F}(1,74)=5.43, \mathrm{p}<0.05]$. Post hoc analyses demonstrated that males exposed to ethanol on G12 showed significant decreases in the frequency of social investigation and play fighting relative to their control counterparts that had been prenatally exposed to saline. Intriguingly, social enrichment had a negative effect on social investigation and play fighting of saline-exposed (but not ethanol-treated) males (fig. 1), with significant decreases in the frequency of play fighting and social investigation seen in control males, but not control females, that underwent social enrichment relative to their nonenriched counterparts.

Contact behavior differed as a function of social enrichment only $[\mathrm{F}(1,74)=5.57, \mathrm{p}<0.05]$, with socially enriched animals demonstrating less contact behavior than their nonenriched counterparts, this effect was driven by males (fig. 1).

The analysis of the coefficient of social preference/ avoidance revealed a significant prenatal treatment $\times$ postnatal housing interaction $[\mathrm{F}(1,74)=8.28, \mathrm{p}<0.01]$. Therefore, post hoc comparisons were done on the data collapsed across sex (fig. 1). Social motivation was significantly decreased in animals exposed to ethanol on G12. This finding is similar to those described previous- 
ly [9]. Social enrichment significantly increased social motivation in the ethanol-exposed animals, thereby mitigating the observed social deficit regardless of sex (fig. 1). Locomotor activity in the social context was not affected by prenatal treatment or postnatal housing (data not shown).

\section{Molecular Analyses}

Of the more than 660 genes examined with involvement in neurotransmission or brain function, we found only a small subset with significant main effects of ethanol exposure at G12 or postnatal social enrichment. Specifically, 10 genes showed significant ethanol effects across sexes and brain areas, and no significant interaction of sex $\times$ ethanol exposure or area $\times$ ethanol exposure (table 1). Interestingly, the majority of these genes showed nominally significant interactions $(\mathrm{p}<0.05, \mathrm{n}=$ 7) or trends for interactions $(\mathrm{p}<0.10, \mathrm{n}=2)$ of ethanol $\times$ enrichment, indicating that the change produced by one treatment could be opposite that produced by the other. Similarly, 20 genes showed significant effects of social enrichment across sexes and brain areas, and no significant interaction of sex $\times$ enrichment or area $\times$ enrichment (table 2). Of note, 2 genes (Chrna6 and Rabep2) showed significant effects of both ethanol exposure and social enrichment and were also the only genes in table 2 that displayed nominally significant interactions of ethanol $\times$ enrichment, indicating that the effects due to enrichment likely differed according to whether an animal was exposed to ethanol or not and vice versa.

Fig. 1. Changes in social behavior following prenatal ethanol exposure and reversal with social enrichment. Prenatal exposure to ethanol on G12 negatively affected play fighting and social investigation in male offspring at P42 (top left). These effects were not seen in females (right). Social enrichment negatively affected play fighting and social investigation in saline-exposed male but not female rats. Social motivation (bottom) was negatively affected in both males and females following prenatal ethanol exposure. Social enrichment (grey bars) improved social motivation in ethanol-exposed animals. For play fighting and social investigation: ${ }^{a} \mathrm{p}<0.05$, significant differences relative to same-sex saline-exposed nonenriched animals; ${ }^{b} \mathrm{p}<0.05$, significant differences relative to same-sex saline-exposed enriched animals. For social motivation: ${ }^{c} p<0.05$, significant differences relative to salineexposed nonenriched animals; ${ }^{d} \mathrm{p}<0.05$, significant differences relative to ethanol-exposed nonenriched animals, with post hoc comparisons performed on the data collapsed across sex. Bars are the mean for each group, $t$ bars show the standard error of the mean. $\mathrm{n}=9-12$ per group.
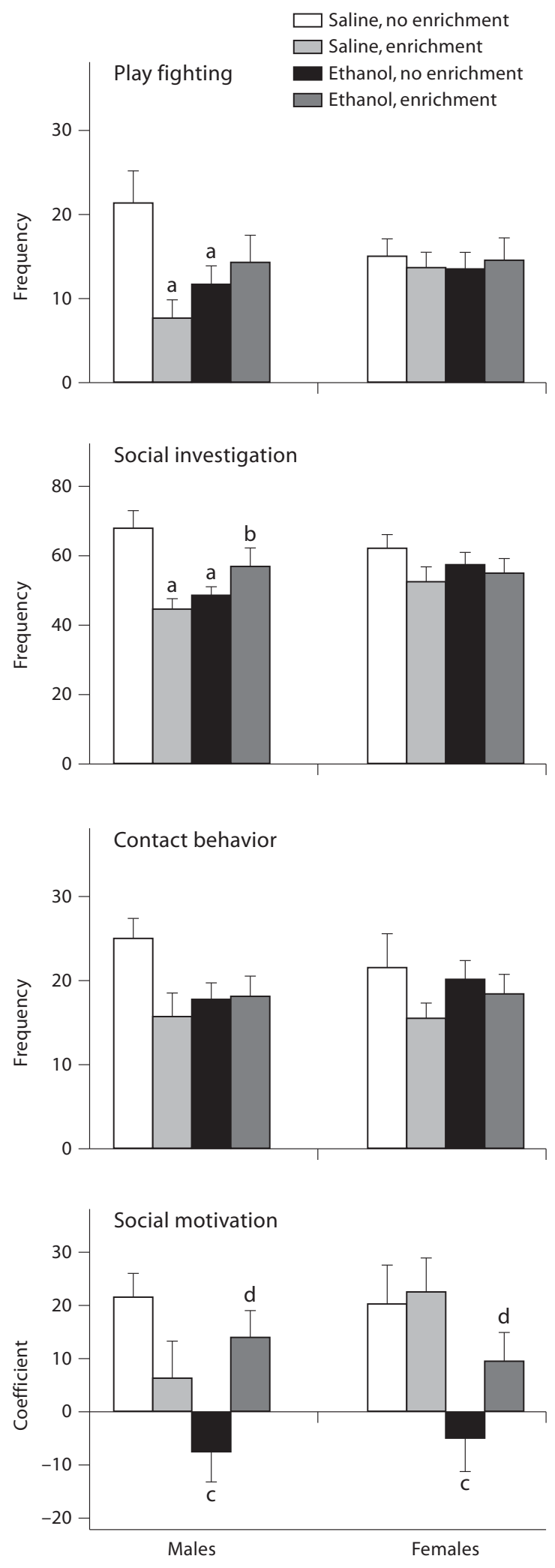


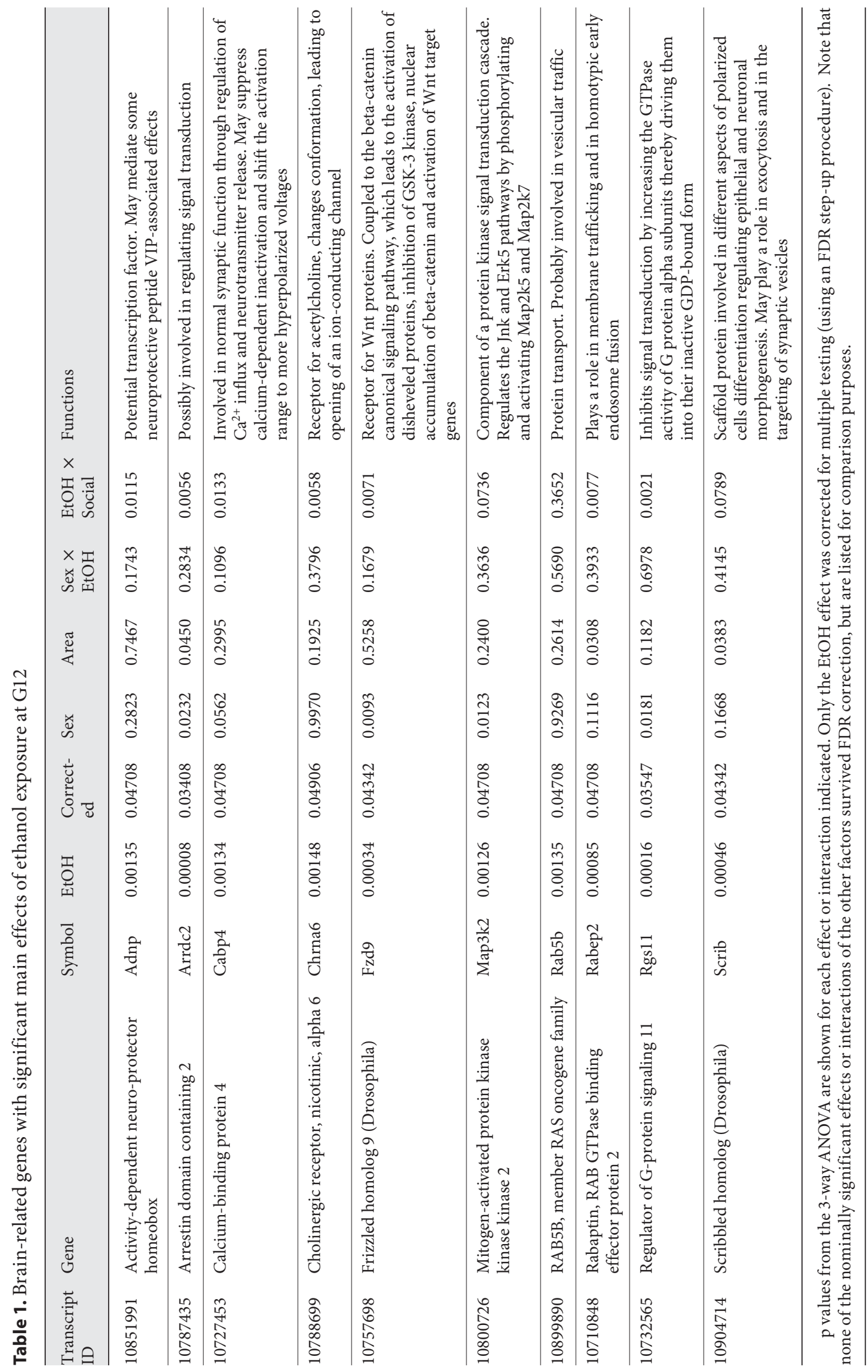




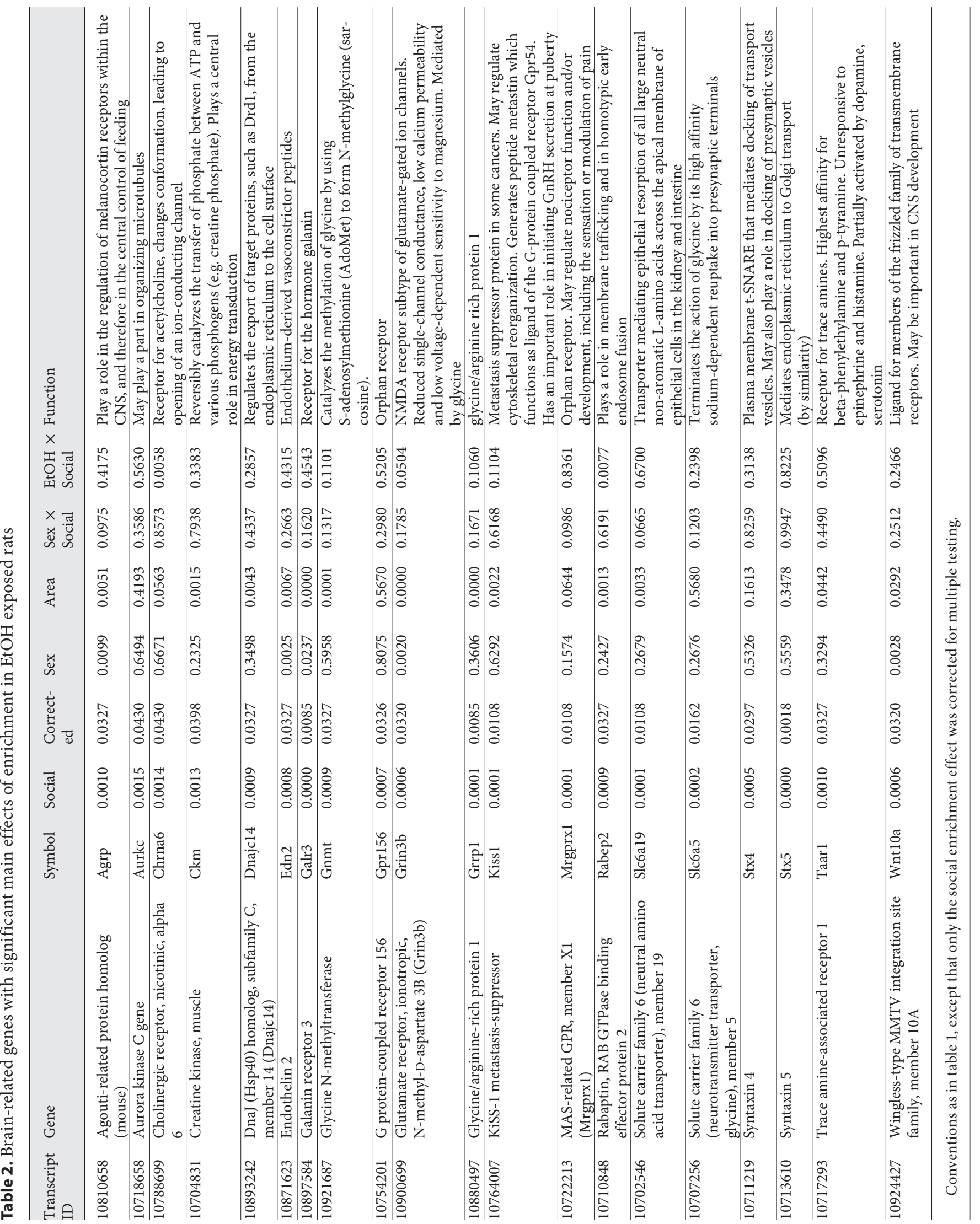




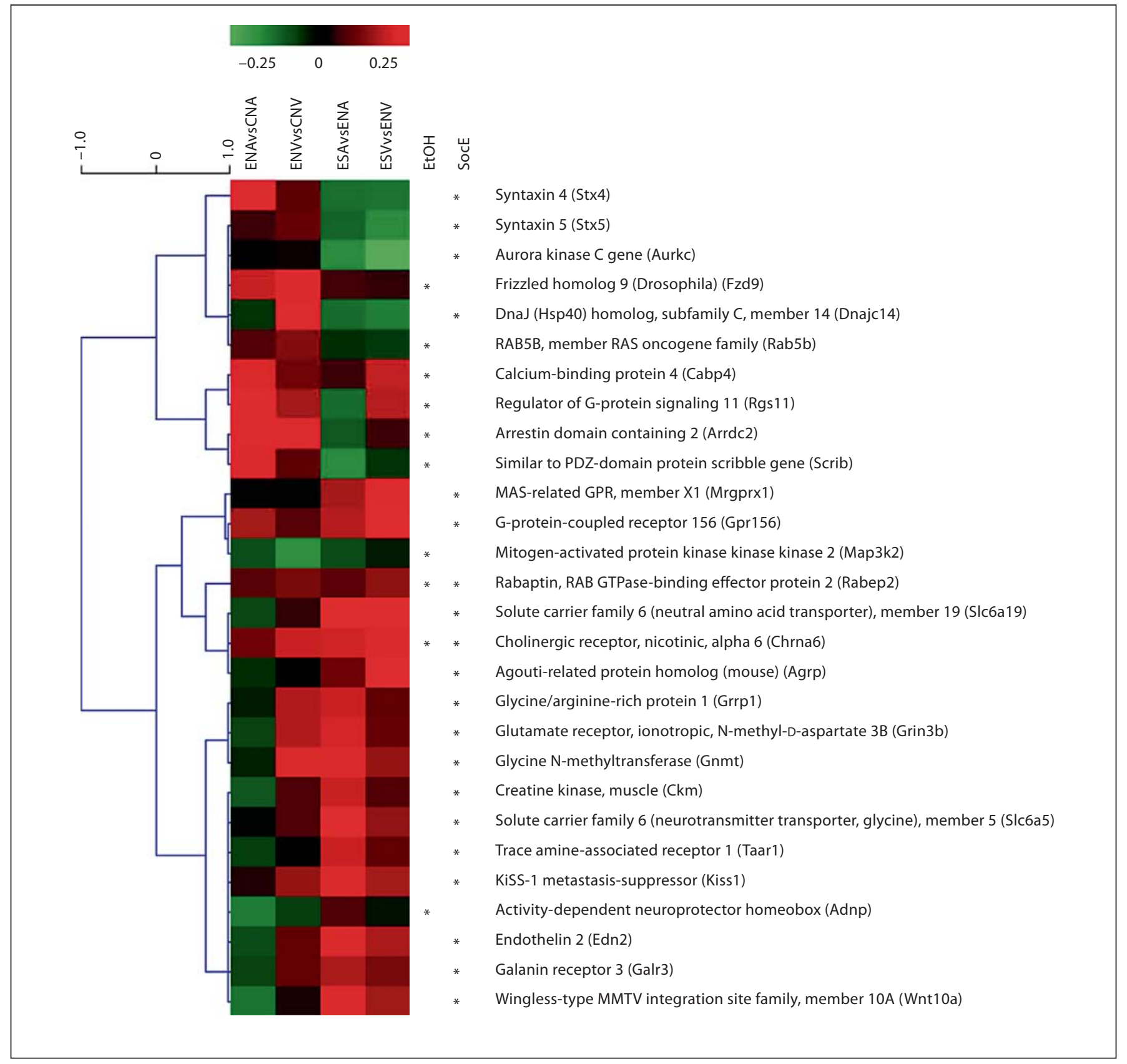

Fig. 2. Hierarchical cluster analysis of brain-related genes with significant effects of gestational ethanol exposure or postnatal social enrichment. The similarity between genes (as measured by a tau coefficient) is indicated by the blue dendrogram below the coefficient scores. The two leftmost colored columns indicate the $\log 2$ change in expression (scale bar shown at the top) in ethanolexposed male and female rat samples versus control rats for the amygdala (ENAvsCNA) and ventral striatum (ENVvsCNV). The two rightmost colored columns indicate the changes in ethanolexposed male and female rats that underwent social enrichment versus no enrichment for the amygdala (ESAvsENA) and the ventral striatum (ESVvsENV). Genes with significant main effects of the ethanol exposure (EtOH) and/or social enrichment (SocE) are indicated by the asterisks before their names. Note that of the genes with a significant EtOH effect, most showed increases in expression (red) in both brain areas, with the notable exceptions of Map3k2 and Adnp. The same genes generally showed trends for decreases in expression (green) in the SocE comparison. The same reciprocal relationship was generally the case for those genes with a significant main effect of social enrichment, although the trends were somewhat less consistent across both brain areas in the EtOH sample groups. Interestingly, two genes (Chrna6 and Rabep2) showed significant main effects of both ethanol and social enrichment, and the direction of changes produced by those treatments was the same in both cases (increased expression). 


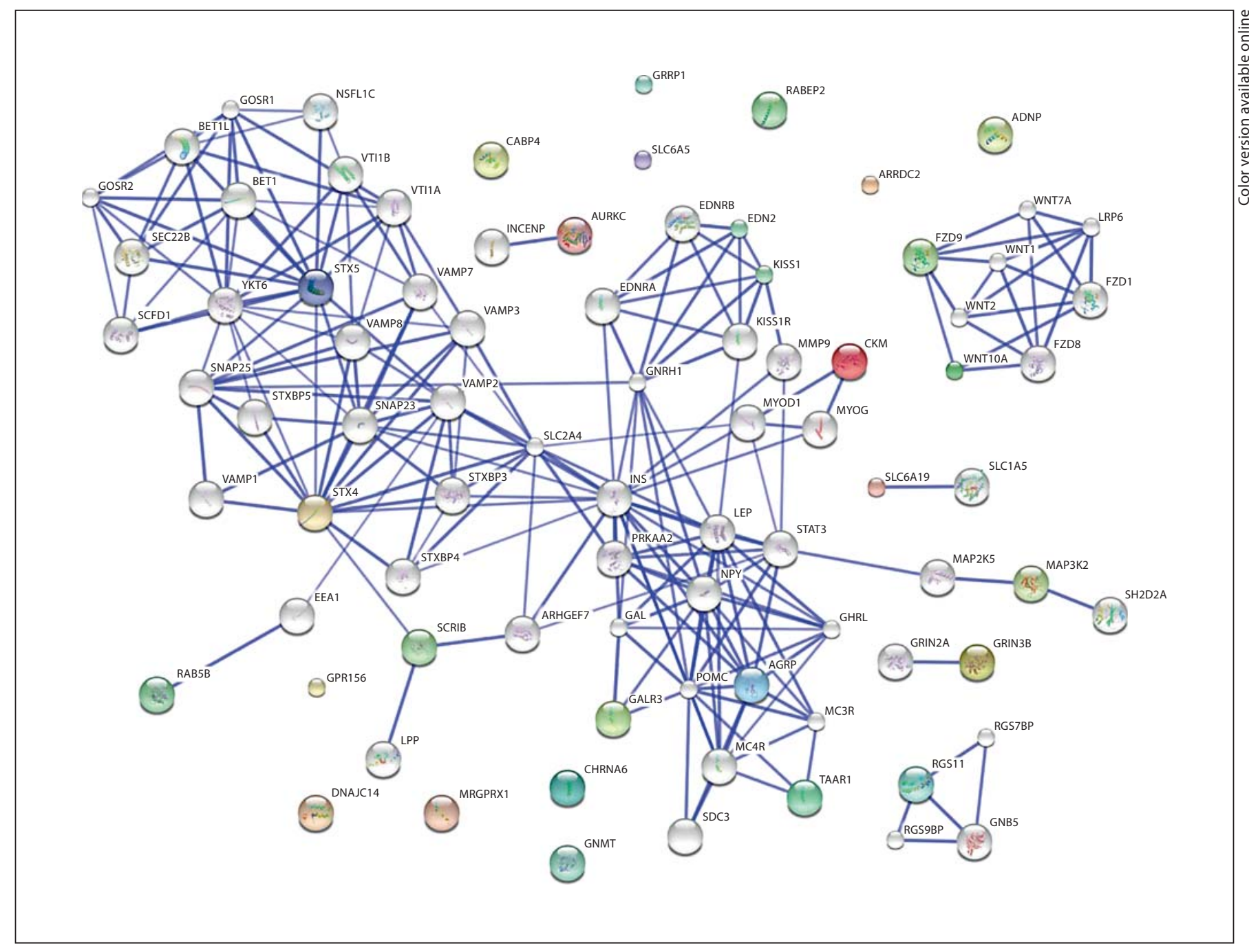

Fig. 3. Biological interaction network for genes with ethanol or social enrichment effects. The 28 total genes identified in the microarray screen were subjected to functional mapping using the STRING database. These genes are indicated with colored spheres. An additional 56 of connecting gene nodes were added in to facilitate detection of interaction networks (genes indicated with white spheres). The strength of the interactions between proteins is indicated by the thickness of the lines interconnecting them. We note that only about half the genes of interest were strongly interconnected with other genes, while several of the genes displayed no identified interactions.
Hierarchical cluster analysis was used to visualize the patterns of expression changes for all of the genes in tables 1 and 2 . We observed that of the 10 genes with a significant ethanol effect, most showed increases in expression in both brain areas, with the notable exceptions of Map3k2 and Adnp (fig. 2). Interestingly, the same genes generally showed trends for decreases in expression as a result of social enrichment. The same reciprocal relationship was often the case for those genes with a significant main effect of social enrichment, although the trends were somewhat less consistent across both brain areas in the etha- nol-exposed sample groups. We note that the two genes with significant main effects of both, ethanol and social enrichment (Chrna6 and Rabep2), produced changes largely in the same direction in both comparisons (increased expression) when comparisons were made within separate groups (ethanol-unenriched vs. control-unenriched, and ethanol-enriched vs. ethanol-unenriched; fig. 2). Interestingly, the nominally significant ethanol $x$ enrichment interaction that was detected for these genes was due to the fact that comparisons of the control-enriched versus control-unenriched samples (not shown) re- 


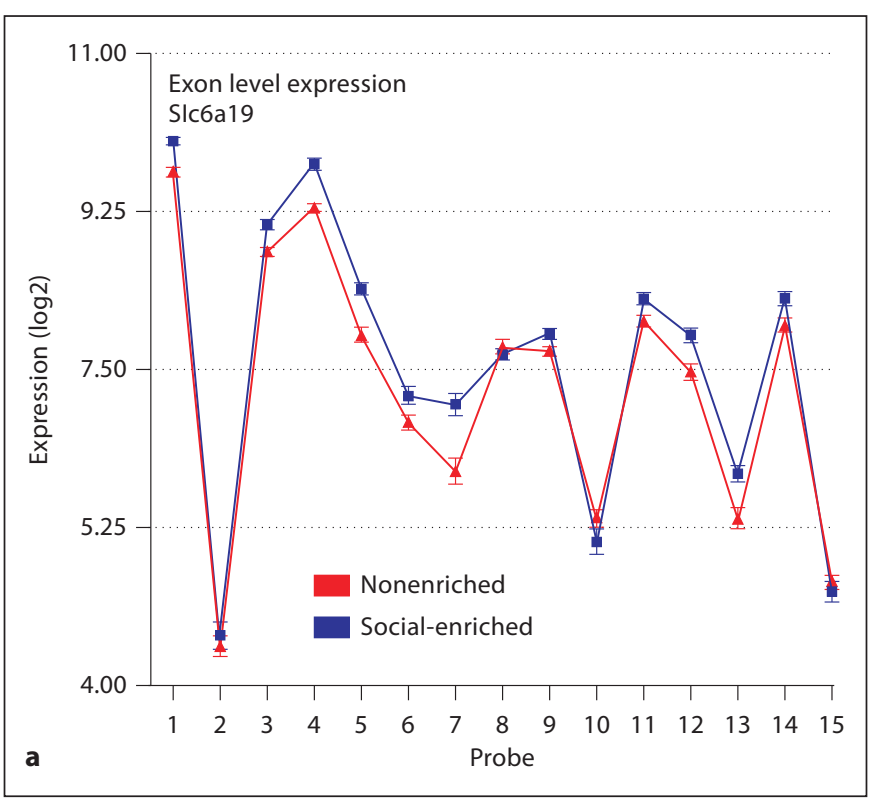

Fig. 4. Significant alternative splicing effects on genes with significant social enrichment effects in G12 ethanol-exposed rats. The solute carrier family 6 (neutral amino acid transporter), member 19 (Slc6a19; a) and KiSS-1 metastasis-suppressor (Kiss1; b) genes showed the two most significant alternative splicing effects ( $p=0.0007$ and 0.0008 , respectively) of all the genes with

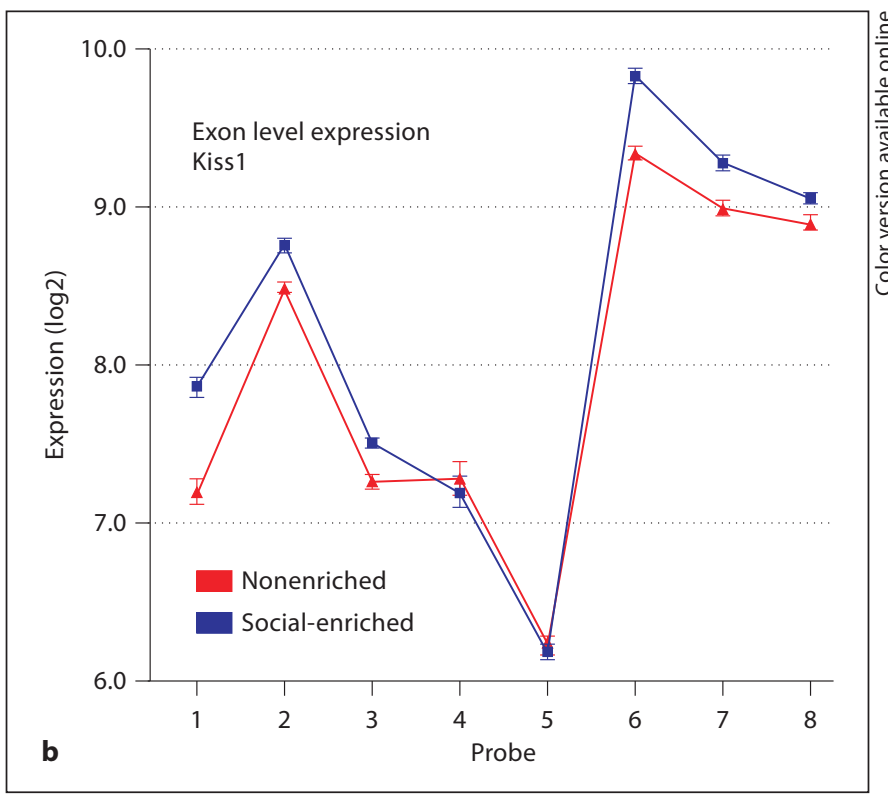

significant main effects on overall gene expression. The $\log 2$ expression of each exon (and standard error bars) are shown. Notably, Slc6a19 tended to show differential enrichment of exons in the middle of the transcript cluster, while Kiss1 showed differential enrichment of exons in the $5^{\prime}$ and $3^{\prime}$ ends of the transcript cluster. $\boldsymbol{\Delta}$ = Nonenriched; $\mathbf{\square}$ = social-enriched. vealed changes that were more than twice as large as those seen in the other comparisons. Thus, although ethanol increased expression of these genes, social enrichment increased expression to a much greater extent, producing a statistical interaction due to differences in the magnitude of the effect produced by each treatment.

A biological interaction network was constructed using all 28 unique genes that we identified as input for the STRING database (fig. 3). Approximately half the genes were found to strongly interact with other proteins that were at least distantly connected, while the other half of the genes were largely solitary. Four subclusters were identified that interconnected (1) Fzd9 and Wnt10a, (2) Stx4, Stx5, Scrib and Rab5b, (3) Kiss1 and Edn2, and (4) Galr3, Agrp, and Taar1.

Finally, we tested all 28 genes with significant ethanol or social enrichment effects for evidence of ASV effects. Interestingly, no ASV effects were seen in the 10 genes with significant prenatal ethanol effects. However, several of the genes with significant effects of social enrichment in ethanol-exposed rats did show putative (nominally significant) splice variant effects. The most significant of these were for the Slc6a19 and Kiss1 genes ( $p=$
0.0007 and 0.0008 , respectively). Examination of the expression level of each exon across the gene indicated consistently increased exon expression for both genes as a consequence of social enrichment. Notably, Slc6a19 showed the greatest increases in the middle of the transcript cluster, while Kiss1 showed the greatest increases in the $5^{\prime}$ and $3^{\prime}$ ends of the cluster (fig. 4).

\section{Discussion}

In this study, we examined whether a form of social enrichment, namely housing rats that had been prenatally exposed to ethanol with peers that had regular prenatal and postnatal experiences, could ameliorate the social deficits seen in the ethanol-exposed rats and what the molecular mechanisms of such effects might be. We found that this particular form of social enrichment normalized the social motivation deficit (i.e., avoidance phenotype) normally seen when they are housed with sex-matched prenatal ethanol-exposed littermates. There were no significant improvements of the other behaviors examined. Molecular analyses of the amygdala and ventral striatum 
of the postnatal rats at $\mathrm{P} 42$ indicated several specific gene expression changes that might contribute to the social avoidance phenotype as well as its reversal.

\section{Social Behavior}

As in our previous study [9], male rats prenatally exposed to ethanol on G12 and housed with littermates after weaning demonstrated decreases in social investigation, contact behavior, and play fighting. These social deficits were not evident in their female counterparts. In accord with our earlier findings, decreases in social motivation, indexed via a transformation of social preference into social avoidance, were evident in both males and females prenatally exposed to ethanol and housed with littermates. These findings suggest that the social consequences of prenatal exposure to ethanol on G12 are extremely robust and can be replicated. It should be noted that these behavioral outcomes are dependent on administration of ethanol on G12, we have previously reported that injection of ethanol on G7 results in a different behavioral outcome [9]. It is not clear how these findings might be extrapolated to a model in which the ethanol exposure is over a longer period of time. Findings by others show changes in social behavior after administration of ethanol to rats during gestation or gestation plus the early postnatal period $[8,40,41]$. However, each of these studies used a different method of ethanol administration, achieved different blood ethanol concentrations, administered different behavioral tests, and measured different outcomes.

Animals prenatally exposed to ethanol demonstrated social avoidance at $\mathrm{P} 42$, whereas social preference was evident in controls. Our earlier findings have shown that the coefficient of social preference/avoidance is an index of anxiety-like behavior in the social context, since this measure is extremely sensitive to both anxiogenic $[42,43]$ and anxiolytic $[43,44]$ manipulations. Specifically, when tested in an unfamiliar, anxiety-provoking environment, animals demonstrate social avoidance regardless of age and sex. In contrast, testing under familiar, nonstressful circumstances results in social preference [45]. Taken together with the results of the present study, these findings suggest that prenatal exposure to ethanol enhances social anxiety. Intriguingly, this social anxiety can be ameliorated by housing of the socially anxious animals with peers that never experienced ethanol in utero and, as a result, have a 'normal social phenotype' (fig. 1). Our results are in agreement with earlier findings demonstrating that rearing of laboratory rodents in enriched environments reduces anxiety-like behavioral manifestations $[31,46,47]$.

Molecular Substrates of Social Avoidance
Social enrichment, however, was ineffective in mitigating decreases in social interactions seen in males following prenatal ethanol. These findings are in contrast with reports that environmental enrichment can reverse social deficits induced by prenatal insult. For instance, Morley-Fletcher et al. [48] have found that environmental enrichment ameliorated social deficits induced by prenatal stress by substantially increasing social interactions in prenatally stressed animals. Similarly, Schneider et al. [25] have reported that environmental enrichment reversed social deficits induced by prenatal exposure to valproic acid. Enriched animals (housed in groups of 12 in a large cage filled with toys) included both those exposed to valproic acid and unexposed controls, and showed elevated levels of play fighting as adolescents and increased social interactions as adults relative to nonenriched controls.

In contrast to these socially enhancing effects of environmental enrichment seen in control males [25], our socially enriched control males demonstrated reliable decreases in social behavior, an intriguing and unexpected finding. One possible explanation is that our 'socially enriched' control animals were withdrawn from their social milieu, and this separation from littermates on the day of weaning and placement in a cage with nonlittermates was extremely stressful for males and resulted in decreased social interactions later in life. Unfortunately, the effects of housing with littermates versus nonlittermates after weaning on social behavior during adolescence have not been investigated so far.

\section{Gene Expression}

The set of genes that we identified with significant ethanol effects included those with direct involvement in neurotransmitter binding or voltage regulation (Achr6, Cabp4), as well as neurotransmitter/synaptic vesicle transport (Rab5b, Rabep2, Scrib), and second messenger signaling (Rgs11, Arrdc2, Map3k2) (table 1). In contrast, the majority of genes with significant effects of social enrichment in ethanol-exposed rats displayed primary involvement in receptor functions (table 2). One reason for the possible differential effect could be the fact that the ethanol exposure was more temporally remote than the social enrichment.

In many cases, the genes that were significantly affected by ethanol exposure on G12 displayed changes in expression that were reversed by social enrichment (though such changes did not survive correction for multiple testing). The same was also true of the effects produced by social enrichment (fig. 2). However, the two 
genes that were independently changed in both analyses (Chrna6 and Rabep2) showed similar changes (increased expression) as a result of ethanol exposure as well as social enrichment. Notably, these were the only genes significantly affected by ethanol that showed a nominally significant ethanol $\times$ enrichment interaction. In this case, however, the interaction was due to the fact that the increased expression seen with ethanol exposure or seen with social enrichment in ethanol-exposed animals (fig. 2) was less than half the increase seen due to social enrichment alone in animals who were not exposed to ethanol (not shown). Apart from these two genes, whose effects may be nonspecific, we believe that the set of genes we identified may serve as a key molecular network in the amygdala-forebrain circuitry that regulates decreased social motivation seen following prenatal ethanol exposure and increased social motivation seen following subsequent social enrichment. It is beyond the scope of this report to review the functions of all of these genes. However, in the space that follows, we briefly highlight a few of the genes that we identified with more novel roles related to amygdala or social functioning.

Galanin is a 29- or 30-amino acid neuropeptide that functions in a manner similar to a neurotransmitter, and plays an important role in the hypothalamus, where it interacts with other gene products such as Agrp and Pomc (fig. 3), that play well-characterized roles in regulating food intake. Of greater relevance to the current study, galanin has recently been recognized as involved in the regulation of mood and anxiety, the central response to stress, and the regulation of ethanol intake and dependence $[49,50]$. Galanin exerts its effects through one of three receptor subtypes (Galr1, Galr2, Galr3). These receptors exhibit regional and cellular differences in expression [51]. In our studies, we found that Galr3 expression was significantly increased in the amygdala and ventral striatum following social enrichment, while showing trends for region-specific changes following prenatal ethanol exposure (decreased expression in the amygdala, increased expression in the ventral striatum; fig. 2).

Ethanol is known to strongly affect GABAergic transmission. Interestingly, based on slice recordings, Bajo et al. [50] recently determined that Galr3 likely augments GABAergic transmission (enhances inhibitory postsynaptic potentials) in specific populations of neurons in the central nucleus of the amygdala at the postsynaptic level, and that ethanol augments GABAergic transmission at the presynaptic level in those same cells. Together, the effects of ethanol and galanin were additive, resulting in markedly reduced inhibitory postsynaptic potentials in the central nucleus of the amygdala when the compounds were coadministered. Taken together, these findings suggest that the trend toward reduced Galr3 expression in the amygdala in our rats prenatally exposed to ethanol could serve to decrease inhibitory conductance in this structure. In contrast, the significant increases in Galr3 expression in the amygdala (as well as ventral striatum) following social enrichment suggests that these brain structures may be attempting to restore a more 'normal' level of inhibitory control (inhibitory postsynaptic potentials), and possibly reduce the excitatory drive of this structure. These possibilities are further supported by the available data on the use of galanin or galanin receptor agonists in the treatment of rodent models of anxiety and depressive disorders [49]. In fact, Karlsson and Holmes [49] suggested that '... galanin recruitment during extreme behavioral and physiological provocations such as stress and opiate withdrawal may serve to attenuate negative emotional states caused by noradrenergic hyperactivation.'

Another gene with an important novel role in regulating brain function is Kisspeptin (Kiss1). Mature Kiss1 is a 54-amino acid neuropeptide that signals through a Gprotein-coupled receptor and is normally expressed at high level in the arcuate and periventricular regions of the hypothalamus, where it regulates the response of hypothalamic neurons to growth hormones (e.g. Igf1) as well as gonadal hormones and the initiation of puberty [52], in part through stimulation of luteinizing hormonereleasing hormone release. However, outside the hypothalamus, the role of Kiss1 is poorly understood. The expression level of Kiss1 is high in specific regions of the amygdala, including the medial nucleus which has been implicated in social and emotional behavior, and the ventral striatum where its expression may be isoform-specific, and strongly influenced by circulating sex hormones, with highest levels seen during proestrous and lowest levels in both male and female gonadectomized mice and rats [52]. Brain levels of Kiss1 mRNA are also known to decrease in response to stress [53]. In our data on adolescent rats, we found that levels of Kiss1 were significantly increased following social enrichment, but showed no significant change (or only a trend for increased expression in the ventral striatum) following prenatal ethanol exposure (fig. 2). Moreover, we also found evidence to support a possible effect of social enrichment on exon usage (fig. 3). Prenatal ethanol exposure is known to delay pubertal onset and decrease sexual behavior in adolescent rats, at least partly through effects on luteinizing hormone-releasing hormone release [54]. Hiney et al. [55] noted that acute ethanol administration normally blocks 
Igf1-induced luteinizing hormone-releasing hormone release, and tested whether changes in Kiss1 expression modulated these effects. They reported that the Igf1-induced expression of Kiss1 was blocked by ethanol. Thus, their data support our findings of lack of a direct effect of ethanol on Kiss1 mRNA expression, but underscore the ability of ethanol to influence Kiss1-mediated neuroendocrine and behavioral responses. The fact that we observed significantly increased expression of Kiss1 following social enrichment in prenatal ethanol-exposed adolescents suggests that this behavioral treatment could help 'normalize' the delays in sexual maturity and sexual behavior documented in other studies of such rats. Viewed in this context, it is intriguing to speculate about a role of the amygdala in regulating both sexual and nonsexual forms of social behavior.

In addition to Galr3 and Kiss1, several other genes showed patterns of change which at least partly resembled the behavioral data (opposing trends due to ethanol and social enrichment). These genes included three that were increased by ethanol and decreased by social enrichment (Stx4, Stx 5 and Rab5b) and two that were decreased by ethanol and increased by social enrichment (Adnp and Taar1). The first of these sets of genes are notable because of their involvement in vesicular trafficking, including synaptic vesicle docking and fusion as well as endosome recycling of postsynaptic receptors [56]. The second set of genes are notable because Adnp is thought to function as a transcription factor and may regulate $\mathrm{p} 53$, a protein known to be targeted by ethanol [57], and because Taarl specifically modulates dopamine release in the striatum as well as the spontaneous firing of dopaminergic neurons in the ventral tegmental area [58], two structures that are critically involved in addiction and reward-based behavior. Thus, some of the additional genes modified by social enrichment may act through specific intracellular pathways to compensate for the effects produced by prenatal ethanol exposure.

In summary, this study found that social enrichment can reverse the social motivation deficits seen in adolescent rats produced by prenatal ethanol exposure. This effect was present in both male and female rats. Our molecular analysis of neurotransmission-related genes suggested a number of specific genes that could contribute to the deficit and its reversal. Some of these genes, such as galanin and Kiss1, represent intriguing opportunities for future studies of the role of the amygdala and ventral striatum in social behavior.

\section{Acknowledgements}

We thank Renee Mezza, Wendi Burnette, Terri Novak, and Bill Bondi for help with the behavioral testing, and Karen Gentile for RNA purification and microarray processing. This research was supported by the National Institute of Alcohol Abuse and Alcoholism (AA015413 and AA018693 to S.M.M., AA012453 to E.I.V., and AA178231 to S.M.M., F.A.M., and E.I.V.) and Autism Speaks (S.M.M.).

\section{References}

1 Mordre M, Groholt B, Knudsen AK, Sponheim E, Mykletun A, Myhre AM: Is longterm prognosis for pervasive developmental disorder not otherwise specified different from prognosis for autistic disorder? Findings from a 30-year follow-up study. J Autism Dev Disord 2011, E-pub ahead of print.

$\checkmark 2$ Thomas SE, Kelly SJ, Mattson SN, Riley EP: Comparison of social abilities of children with fetal alcohol syndrome to those of children with similar IQ scores and normal controls. Alcohol Clin Exp Res 1998;22:528-533.

$\checkmark 3$ Kelly SJ, Day N, Streissguth AP: Effects of prenatal alcohol exposure on social behavior in humans and other species. Neurotoxicol Teratol 2000;22:143-149.

-4 Roebuck TM, Mattson SN, Riley EP: Behavioral and psychosocial profiles of alcoholexposed children. Alcohol Clin Exp Res 1999;23:1070-1076.

-5 Whaley SE, O'Connor MJ, Gunderson B: Comparison of the adaptive functioning of children prenatally exposed to alcohol to a nonexposed clinical sample. Alcohol Clin Exp Res 2001;25:1018-1024.

-6 Olson HC, Feldman JJ, Streissguth AP, Sampson PD, Bookstein FL: Neuropsychological deficits in adolescents with fetal alcohol syndrome: clinical findings. Alcohol Clin Exp Res 1998;22:1998-2012.

$\checkmark 7$ Meyer LS, Riley EP: Social play in juvenile rats prenatally exposed to alcohol. Teratology $1986 ; 34: 1-7$.

8 Lugo JN Jr, Marino MD, Cronise K, Kelly SJ: Effects of alcohol exposure during development on social behavior in rats. Physiol Behav 2003;78:185-194.

-9 Mooney SM, Varlinskaya EI: Acute prenatal exposure to ethanol and social behavior: effects of age, sex, and timing of exposure. Behav Brain Res 2011;216:358-364.

10 Daenen EW, Wolterink G, Gerrits MA, Van Ree JM: The effects of neonatal lesions in the amygdala or ventral hippocampus on social behaviour later in life. Behav Brain Res 2002; 136:571-582.

11 Diergaarde L, Gerrits MA, Stuy A, Spruijt BM, van Ree JM: Neonatal amygdala lesions and juvenile isolation in the rat: differential effects on locomotor and social behavior later in life. Behav Neurosci 2004;118:298-305.

-12 Spezio ML, Huang PY, Castelli F, Adolphs R: Amygdala damage impairs eye contact during conversations with real people. J Neurosci 2007;27:3994-3997.

13 Adolphs R, Sears L, Piven J: Abnormal processing of social information from faces in autism. J Cogn Neurosci 2001;13:232-240.

14 Adolphs R, Baron-Cohen S, Tranel D: Impaired recognition of social emotions following amygdala damage. J Cogn Neurosci 2002; 14:1264-1274.

-15 Spezio ML, Adolphs R, Hurley RS, Piven J: Abnormal use of facial information in highfunctioning autism. J Autism Dev Disord 2007;37:929-939. 
16 Price JL: Comparative aspects of amygdala connectivity. Ann NY Acad Sci 2003;985: 50-58.

17 Pennartz CM, Ito R, Verschure PF, Battaglia FP, Robbins TW: The hippocampal-striatal axis in learning, prediction and goal-directed behavior. Trends Neurosci 2011;34:548-559.

18 Dawson G, Galpert L: Mothers' uses of imitative play for facilitating social responsiveness and toy play in young autistic children. Dev Psychopathol 1990;2:151-162.

19 Oke NJ, Schreibman L: Training social initiations to a high-functioning autistic child: assessment of a collateral behavioral change and generalization in a case study. J Autism Dev Dis 1990;20:479-497.

20 Stahmer AC: Teaching symbolic play skills to children with autism using pivotal response training. J Autism Dev Disord 1995;25:123142.

-21 Strain PS, Kerr MM, Ragland EU: Effects of peer-mediated social initiations and prompting/reinforcement procedures on social behavior of autistic children. J Autism Dev Disord 1979;9:41-54.

22 Bodfish JW: Treating the core features of autism: are we there yet? Ment Retard Dev Disabil Res Rev 2004;10:318-326

-23 Strain PS: Generalization of autistic children's social behavior change: effects of developmentally integrated and segregated settings. Anal Intervent Dev Disabil 1983;3:23-34.

24 Lewis MH, Tanimura Y, Lee LW, Bodfish JW: Animal models of restricted repetitive behavior in autism. Behav Brain Res 2007;176: 66-74.

25 Schneider T, Turczak J, Przewlocki R: Environmental enrichment reverses behavioral alterations in rats prenatally exposed to valproic acid: issues for a therapeutic approach in autism. Neuropsychopharmacology 2006; 31:36-46.

26 Moser MB, Trommald M, Egeland T, Andersen P: Spatial training in a complex environment and isolation alter the spine distribution differently in rat CA1 pyramidal cells. J Comp Neurol 1997;380:373-381.

-27 Schrijver NC, Bahr NI, Weiss IC, Würbel H: Dissociable effects of isolation rearing and environmental enrichment on exploration, spatial learning and HPA activity in adult rats. Pharmacol Biochem Behav 2002;73:209-224.

28 Tang YP, Wang H, Feng R, Kyin M, Tsien JZ: Differential effects of enrichment on learning and memory function in NR2B transgenic mice. Neuropharmacology 2001;41: 779-790.

-29 Benaroya-Milshtein N, Hollander N, Apter A, Kukulansky T, Raz N, Wilf A, et al: Environmental enrichment in mice decreases anxiety, attenuates stress responses and enhances natural killer cell activity. Eur J Neurosci 2004;20:1341-1347.

-30 Chapillon P, Manneché C, Belzung C, Caston $\mathrm{J}$ : Rearing environmental enrichment in two inbred strains of mice. 1. Effects on emotional reactivity. Behav Genet 1999;29:41-46.

- 31 Friske JE, Gammie SC: Environmental enrichment alters plus maze, but not maternal defense performance in mice. Physiol Behav 2005;85:187-194.

-32 Roy V, Belzung C, Delarue C, Chapillon P: Environmental enrichment in $\mathrm{BALB} / \mathrm{c}$ mice: effects in classical tests of anxiety and exposure to a predatory odor. Physiol Behav 2001; 74:313-320.

33 Pietropaolo S, Branchi I, Cirulli F, Chiarotti F, Aloe L, Alleva E: Long-term effects of the periadolescent environment on exploratory activity and aggressive behaviour in mice: social versus physical enrichment. Physiol Behav 2004;81:443-453.

34 Matthews K, Wilkinson LS, Robbins TW: Repeated maternal separation of preweanling rats attenuates behavioral responses to primary and conditioned incentives in adulthood. Physiol Behav 1996;59: 99-107.

35 Hall FS: Social deprivation of neonatal, adolescent, and adult rats has distinct neurochemical and behavioral consequences. Crit Rev Neurobiol 1998;12:129-162.

36 Weiss IC, Feldon J: Environmental animal models for sensorimotor gating deficiencies in schizophrenia: a review. Psychopharmacology 2001;156:305-326.

37 Würbel H: Ideal homes? Housing effects on rodent brain and behaviour. Trends Neurosci 2001;24:207-211.

38 File SE, Hyde JR: Can social interaction be used to measure anxiety? Br J Pharmacol 1978:62:19-24.

39 File SE, Seth P: A review of 25 years of the social interaction test. Eur J Pharmacol 2003; 463:35-53.

40 Meyer LS, Riley EP: Social play in juvenile rats prenatally exposed to alcohol. Teratology 1986;34:1-7.

41 Hamilton DA, Akers KG, Rice JP, Johnson TE, Candelaria-Cook FT, Maes LI, Rosenberg M, Valenzuela CF, Savage DD: Prenatal exposure to moderate levels of ethanol alters social behavior in adult rats: relationship to structural plasticity and immediate early gene expression in frontal cortex. Behav Brain Res 2010;207:290-304.

42 Doremus-Fitzwater TL, Varlinskaya EI, Spear LP: Social and non-social anxiety in adolescent and adult rats after repeated restraint. Physiol Behav 2009;97:484-494.

43 Varlinskaya EI, Spear LP: Increases in anxiety-like behavior induced by acute stress are reversed by ethanol in adolescent but not adult rats. Pharmacol Biochem Behav 2011; 100:440-450.

44 Varlinskaya EI, Doremus-Fitzwater TL, Spear LP: Repeated restraint stress alters sensitivity to the social consequences of ethanol in adolescent and adult rats. Pharmacol Biochem Behav 2010;96:228-235.

45 Varlinskaya EI, Spear LP: Social interactions in adolescent and adult Sprague-Dawley rats: impact of social deprivation and test context familiarity. Behav Brain Res 2008;188:398405.

46 Fernandez-Teruel A, Escorihuela RM, Castellano B, Gonzalez B, Tobena A: Neonatal handling and environmental enrichment effects on emotionality, novelty/reward seek- ing, and age-related cognitive and hippocampal impairments: focus on the Roman rat lines. Behav Genet 1997;27:513-526.

47 Fernandez-Teruel A, Gimenez-Llort L, Escorihuela RM, Gil L, Aguilar R, Steimer T, Tobena A: Early-life handling stimulation and environmental enrichment: are some of their effects mediated by similar neural mechanisms? Pharmacol Biochem Behav 2002;73:233-245.

48 Morley-Fletcher S, Rea M, Maccari S, Laviola G: Environmental enrichment during adolescence reverses the effects of prenatal stress on play behaviour and HPA axis reactivity in rats. Eur J Neurosci 2003;18:33673374.

49 Karlsson RM, Holmes A: Galanin as a modulator of anxiety and depression and a therapeutic target for affective disease. Amino Acids 2006;31:231-239.

50 Bajo M, Madamba SG, Lu X, Sharkey LM, Bartfai T, Siggins GR: Receptor subtype-dependent galanin actions on gamma-aminobutyric acidergic neurotransmission and ethanol responses in the central amygdala. Addict Biol 2011, E-pub ahead of print.

51 Burazin TC, Larm JA, Ryan MC, Gundlach AL: Galanin-R1 and -R2 receptor $m R N A$ expression during the development of rat brain suggests differential subtype involvement in synaptic transmission and plasticity. Eur J Neurosci 2000;12:2901-2917.

52 Kim J, Semaan SJ, Clifton DK, Steiner RA, Dhamija S, Kauffman AS: Regulation of Kiss1 expression by sex steroids in the amygdala of the rat and mouse. Endocrinology 2011;152:2020-2030.

53 Kinsey-Jones JS, Li XF, Knox AM, Wilkinson ES, Zhu XL, Chaudhary AA, Milligan SR, Lightman SL, O’Byrne KT: Down-regulation of hypothalamic kisspeptin and its receptor, Kiss1r, mRNA expression is associated with stress-induced suppression of luteinising hormone secretion in the female rat. J. Neuroendocrinology 2009;21:20-29.

54 Creighton-Taylor JA, Rudeen PK: Prenatal ethanol exposure and opiatergic influence on puberty in the female rat. Alcohol 1991;8: 187-191.

55 Hiney JK, Srivastava VK, Les Dees W: Insulin-like growth factor-1 stimulation of hypothalamic KiSS-1 gene expression is mediated by Akt: effect of alcohol. Neuroscience 2010; 166:625-632.

56 Mohanasundaram P, Shanmugam MM: Role of syntaxin 4 in activity-dependent exocytosis and synaptic plasticity in hippocampal neurons. Sci Signal 2010;19:3.

57 Wang LL, Yang AK, He SM, Liang J, Zhou ZW, Li Y, Zhou SF: Identification of molecular targets associated with ethanol toxicity and implications in drug development. Curr Pharm Des 2010;16:1313-1355.

58 Lindemann L, Meyer CA, Jeanneau K, Bradaia A, Ozmen L, Bluethmann H, Bettler B, Wettstein JG, Borroni E, Moreau JL, Hoener MC: Trace amine-associated receptor 1 modulates dopaminergic activity. J Pharmacol Exp Ther 2008;324:948-956. 